\title{
NEUROMUSCULAR DISEASES
}

\section{POLGI MUTATIONS AND CHARCOT-MARIE-TOOTH DISEASE}

A 35-year-old man first diagnosed with autosomal recessive Charcot-Marie-Tooth disease type 2 at 22 years of age had an abnormal gait and pes cavus at age 10 years. Molecular analysis of the POLG1 (polymerase-g-1) gene in muscle biopsy showed 3 heterozygous mutations, in a study at Queen's Hospital, Romford; Newcastle University; and other centers in the UK. At 22 years, he had bilateral distal weakness and wasting of the extremities, clawing of toes, tremor of hands, loss of joint position and vibration sense, and nerve conduction studies consistent with axonal sensorimotor neuropathy. At 35 years, he was unable to walk and had developed a prominent no-no head tremor, upbeat and pendular nystagmus, cerebellar dysarthria and dysphagia, postural and action tremor, dysmetria, distal limb weakness and wasting, absent reflexes, and diminished sensation to ali 4 modalities. Other affected family members include a brother and a sister who developed symptoms at 9 years. Muscle histochemistry findings showed denervation and reinnervation, and a mosaic defect of cytochrome c oxidase. Polymerase chain reaction of skeletal muscle DNA revealed multiple deletions of mtDNA. Segregation analysis in the family showed that heterozygous substitutions in the proband POLGl gene sequencing were inherited from the mother and father. The affected brother had the same genotype as that of the proband. (Harrower T, Stewart JD, Hudson G et al. POLGl mutations manifesting as autosomal recessive axonal Charcot-Marie-Tooth disease. Arch Neurol Jan 2008;65:133-136). (Respond: Patrick F Chinnery PhD, FRCP, Mitochondrial Research Group, The Medical School, Newcastle University, Room M41014, Framlington Place, Newcastle upon Tyne NE2 4HH, England; E-mail: p.f.chinnery@ncl.ac.uk).

COMMENT. POLG1 mutations cause deletions and depletions of mtDNA, leading to a respiratory chain defect with organ dysfunction and the clinical phenotype of CMT. This case report and family are unusual since all 3 siblings had signs of peripheral neuropathy only, without signs of multisystem mitochondrial disorder. Tremor and ataxia developed late, without the ophthalmoplegia that is characteristic of the mitochondrial phenotype of sensory ataxic neuropathy with dysphagia and ophthalmoplegia (SANDO). The authors recommend that $P O L G I$ should be sequenced in patients with unexplained CMT, even in the absence of signs of mitochondrial disease.

Two novel connexin32 mutations cause early onset $\mathrm{X}$-linked CMT in 2 Norwegian families. (Braathen GJ, et al. BMC Neurology 2007;7:19-28).

\section{NEUROPATHY AND OTHER NEUROLOGICAL DISORDERS IN GAUCHER DISEASE TYPE 1}

Neurological manifestations of 31 patients with Gaucher disease type 1 (GD1) were evaluated in a study at Miguel Servet University Hospital, Zaragoza, Spain. Twelve were males and 19 females, mean age 39 years (range 5-77). Age at diagnosis of GD was in early childhood or adolescence in 13 
$(42 \%)$ patients. Nerve conduction studies performed in 15 GD1 patients showed abnormalities in 12 ( 2 children); $26-40 \%$ showed reduced amplitude or abnormal wave forms in one to 3 nerves. Motor nerve conduction was normal, but sensory nerve conduction amplitude was reduced in $26 \%$ patients. Twentytwo patients receiving enzyme replacement therapy for 5-12 years showed neurological manifestations in 9. In adults, these were sensory neuropathy, parkinsonism, dementia, cognitive impairment and stroke; and in children, hypoacusia, myoclonus, and psychomotor delay. (Capablo JL, de Cabezon AS, Fraile $J$ et al. Neurological evaluation of patients with Gaucher disease diagnosed as type 1. J Neurol Neurosurg Psychiatry February 2008;79:219222). (Respond: Dr P Giraldo, Servicio de Hematolgia, Hospital Universitario Miguel Servet, Paseo Isabel La Catolica 1-3, 50006 Zaragoza, Spain).

COMMENT. Gaucher's disease, characterized by cerebroside storage in the reticuloendothelial system, occurs in 3 forms: Type 1) chronic GD, slowly progressive with visceral but rarely nervous system involvement; type 2) infantile GD with rapidly progressive CNS disease; and type 3) juvenile GD, with slowly progressive hepatosplenomegaly, intellectual deterioration, cerebellar ataxia, myoclonic seizures, and spasticity. An adult chronic GD is more common and may present in infancy, with rare CNS involvement. The above study shows that neurological abnormalities, including subclinical neuropathy, occasionally present in childhood GD1, and the classification of GD should be considered as a phenotype continuum.

\section{DEMYELINATING DISEASES}

\section{SEROPREVALENCE OF NEUROMYELITIS OPTICA-IgG OF CHILDHOOD COMPARED TO ADULTS}

The clinical and radiological characteristics and serostatus of neuromyelitis optica (NMO)-IgG in 87 children with inflammatory demyelinating CNS disorders were analyzed in a study at the Mayo Clinic, Rochester, MN, and other centers in the US, Canada, and Argentina. Seventeen patients had NMO and of these, 8 (47\%) were seropositive. The prevalence of seropositivity was higher with relapsing NMO (7 of $9,78 \%$ ) than monophasic NMO ( 1 of $8,12.5 \%, p=0.01)$. The majority of children with NMO (14 of 17) were enrolled from the program in Argentina, and few came from Canada. None showed oligoclonal bands in the CSF. MRI abnormalities were revealed in $9(53 \%)$. After a follow-up of 36 months (range 1.2-126 months), $6.3 \%$ children with $\mathrm{NMO}$ were wheelchair-bound and $23 \%$ had severe visual impairment. One of 5 children with relapsing optic neuritis and none of 8 with monophasic optic neuritis was seropositive. Among 41 with relapsing-remitting multiple sclerosis, 9 with transverse myelitis, and 3 with ADEM, none was seropositive. The frequency of NMO-IgG in children is similar to that in adults. Longitudinally extensive 